\title{
LncRNA CASC15 promotes colon cancer cell proliferation and metastasis by regulating the miR-4310/LGR5/Wnt/ $\beta$-catenin signaling pathway
}

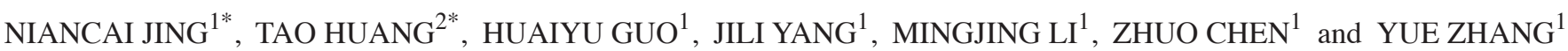 \\ ${ }^{1}$ Department of Integrated Traditional and Western Medicine, Tumor Hospital of Jilin Province, Changchun, Jilin 130012; \\ ${ }^{2}$ Department of Emergency, Affiliated Hospital of Changchun University of Traditional Chinese Medicine, \\ Changchun, Jilin 130000, P.R. China
}

Received January 31, 2018; Accepted June 6, 2018

DOI: $10.3892 / \mathrm{mmr} .2018 .9191$

\begin{abstract}
Previous studies have indicated that overexpression of long noncoding RNA cancer susceptibility 15 (CASC15) may promote tumor development and progression in gastric cancer and hepatocellular carcinoma. However, the function of CASC15 in colon cancer remains unknown. In the present study, the expression of CASC15 was upregulated in colon cancer tissues and its expression was correlated with clinical Tumor-Node-Metastasis stage and tumor metastasis. In addition, knockdown of CASC15 significantly inhibited the proliferation, migration and invasion of colon cancer cells in vitro and in vivo. Following mechanistic experiments, CASC15 was observed to act as a sponge to suppress microRNA (miR)-4310 that targeted LGR5. Through the inhibition of miR-4310, CASC15 promoted leucine-rich repeat-containing G-protein coupled receptor 5 (LGR5) expression and consequently activated the $\mathrm{Wnt} / \beta$-catenin signaling pathway. The results revealed that the inhibition of the $\mathrm{Wnt} / \beta$-catenin signaling pathway in CASC15-overexpressing colon cancer cells suppressed cellular proliferation, migration and invasion. Collectively, these results demonstrated that CASC15 promoted colon cancer growth and metastasis through the activation of the $\mathrm{Wnt} / \beta$-catenin signaling pathway in a miR-4310/LGR5 dependent manner. Thus, the present study suggested that CASC15 may be a therapeutic target for colon cancer treatment.
\end{abstract}

Correspondence to: Professor Yue Zhang, Department of Integrated Traditional and Western Medicine, Tumor Hospital of Jilin Province, 1018 Huguang Road, Changchun, Jilin 130012, P.R. China

E-mail: zhuangyue1653@163.com

${ }^{*}$ Contributed equally

Key words: long noncoding RNA, microRNA, cancer susceptibility 15 , colon cancer, proliferation

\section{Introduction}

Colon cancer (CC) is one of the most common cancers and leads to most cancer-related deaths around the world every year (1). The incidence and mortality rates of CC are still increasing and $\mathrm{CC}$ has become a major public health problem worldwide (2,3). Although some advance has been achieved on the treatment of $\mathrm{CC}$ in recent years, the five-year survival rate of CC patients still remains very low (4). Therefore, there is an urgent requirement to develop new and effective therapeutic strategies. And the molecular mechanism underlying tumorigenesis of $\mathrm{CC}$ needs to be thoroughly investigated.

Long noncoding RNAs (lncRNAs) are a member of noncoding RNA and possess a length of over 200 nucleotides, but have no protein-coding potential (5-8). Many studies show that IncRNA has widely functions in a diversity of biological processes, such as development, cell proliferation, apoptosis and mobility (9-13). LncRNA has been demonstrated to be involved in the development and progression of various cancers (14). Abnormal expression of lncRNAs is often observed in cancers, including CC (15). For instance, lncRNA Igf2as controls hepatocellular carcinoma progression through the ERK/MAPK signaling pathway (16). LncRNA BLACAT1 indicates a poor prognosis of colorectal cancer and affects cell proliferation by epigenetically silencing of p15 (17). LncRNA CCAT2 promotes tumorigenesis by over-expressed Pokemon in non-small cell lung cancer (18). Previous evidence indicates that IncRNA RNA cancer susceptibility 15 (CASC15) is upregulated in hepatocellular carcinoma and facilitates hepatocarcinogenesis (19). However, the function of CASC15 in CC is still largely unknown.

In this study, we aimed to investigate the role of CASC15 in $\mathrm{CC}$ and explain its functional mechanism. We first found that the expression of CASC15 was upregulated in CC tissues and cell lines, and knockdown of CASC15 significantly inhibited the proliferation, migration and invasion of $\mathrm{CC}$ cells in vitro and in vivo. In term of mechanism, we found that CASC15 overexpression activated the $\mathrm{Wnt} / \beta$-catenin pathway by promoting G-protein coupled receptor 5 (LGR5) expression through sponging microRNA (miR)-4310. What's more, inhibition of $\mathrm{Wnt} / \beta$-catenin pathway 
significantly inhibited the proliferation, migration and invasion of CASC15-overexpressing CC cells. Taken together, our findings demonstrated the cancer-promoting role of CASC15 in $\mathrm{CC}$ via activation of $\mathrm{Wnt} / \beta$-catenin pathway.

\section{Materials and methods}

Human samples. The fresh clinical CC tissues and adjacent normal tissues were obtained from patients who underwent surgical treatment for CC at Affiliated Hospital of Changchun University of Traditional Chinese Medicine (Changchun, China) between August 2014 and December 2016. All tissues were immediately placed in liquid nitrogen in a freezing tube following surgery and were stored at $-80^{\circ} \mathrm{C}$ until the extraction of total RNA and protein were performed. All experiments in the study were performed in accordance with guidelines and regulations, and were approved by the Institutional Ethics Committee of Tumor Hospital of Jilin Province (Changchun, China); written informed consent was obtained from all participants. None of the patients received preoperative chemotherapy or radiotherapy. All samples were blindly confirmed by two experienced pathologists. Clinical stage was conducted in accordance with the protocols of the American Joint Committee on Cancer Staging System.

Cell lines and cell culture. The human colon cancer cell lines (HT29, HCT116, SW480, SW620, and LoVo), and the human normal colonic epithelial cell line (NCM460) were purchased from the American Type Culture Collection (ATCC, Manassas, VA, USA). All cells were cultured in Roswell Park Memorial Institute 1640 medium (RPMI-1640) supplemented with $10 \%$ fetal bovine serum (FBS; both Gibco; BRL, Thermo Fisher Scientific, Inc., Waltham, MA, USA), $100 \mathrm{U} / \mathrm{ml}$ penicillin, and $100 \mathrm{mg} / \mathrm{ml}$ streptomycin (Sigma-Aldrich; Merck KGaA, Darmstadt, Germany) at $37^{\circ} \mathrm{C}$ in a humidified incubator containing $5 \% \mathrm{CO}_{2}$.

Cell transfection. A short hairpin RNA (shRNA)-targeted CASC15 was designed by GenePharma (Shanghai, China) and cloned into pRNAT-U6.1/Neo plasmid (Biovector, Beijing, China) to generate a pRNAT-U6.1/Neo-shCASC15 plasmid. The empty pRNAT-U6.1/Neo plasmid was used as the negative control (shNC). All constructs were confirmed by DNA sequencing. To knockdown CASC15 expression, CC cells were transfected with shNC or shCASC15 by using Lipofectamine 2000 (Invitrogen; Thermo Fisher Scientific, Inc.) in accordance with the manufacturer's instructions. For establishing a cell line with stable silence of CASC15, the plasmids carrying shCASC15 or shNC were co-transfected with packaging vectors, namely, pMDLg/pRRE, pRSV-REV, and pCMV-VSVG, to produce pseudotyped lentiviruses designated as Lv-shSUMO1P3-1 and Lv-shNC. The lentiviruses were concentrated by ultracentrifugation and then infected CC cells. After 2 weeks of screening using $200 \mu \mathrm{g} / \mathrm{ml}$ neomycin (Sigma-Aldrich; Merck KGaA) for CC cells transfected with shCASC15 or shNC plasmids (in vitro assays) single clones were collected.

Tumorigenesis assay in vivo. Five-week-old male athymic nude $\mathrm{BALB} / \mathrm{c}$ mice were maintained under specific pathogen-free conditions and manipulated according to protocols approved by the Medical Experimental Animal Care Commission at Tumor Hospital of Jilin Province; all experimental procedures were conducted in accordance with the Guide for the Care and Use of Laboratory Animals. For subcutaneous xenograft assay, $5 \times 10^{5} \mathrm{SW} 480$ cells infected with shNC or shCASC15 were subcutaneously inoculated in the flanks of the mice ( $\mathrm{n}=10$ mice/group). A caliper was used to examine the tumor volumes once a week. At 5 weeks post-inoculation, the mice were sacrificed by euthanasia. The tumors were then removed and weighed.

Cell proliferation assays. 2000 HCT-116 or SW480 cells were seeded in 96-well plates for 3 days. Cell Counting kit-8 (CCK8) (Beyotime, Shanghai, China) was used to assess the cell proliferation. In general, $10 \mu \mathrm{l}$ CCK8 solution was added to each plate and cells were incubated for $2 \mathrm{~h}$ in $37^{\circ} \mathrm{C}$. The cell viability was revealed by the absorbance which was measured at $450 \mathrm{~nm}$.

In vitro migration and invasion assay. SW480 and HCT-116 cells transfected with shCASC15 were harvested after $48 \mathrm{~h}$. The cells were resuspended in serum-free medium, and $3 \times 10^{4}$ cells $(100 \mu \mathrm{l})$ was plated into the upper chambers of Transwell inserts $(8.0-\mu \mathrm{m}$ pore size; Corning Inc., Corning, NY, USA) for invasion (with Matrigel) or migration (without Matrigel) assays. The inserts were placed in 24-well plates containing $600 \mu 1$ media with $10 \%$ FBS as a chemoattractant. After incubation for $24 \mathrm{~h}$ for migration and $48 \mathrm{~h}$ for invasion, cells were fixed with $4 \%$ triformol for $20 \mathrm{~min}$ and stained with $1 \%$ crystal violet. The cell numbers were calculated and imaged under a microscope from five random fields.

Cell cycle analysis by flow cytometry. The CC cells were trypsinized and washed with cold phosphate-buffered saline (PBS). The cells were then fixed with ice-cold $70 \%$ ethanol at $4^{\circ} \mathrm{C}$ overnight. After washing with PBS, the cells were treated with RNAase (Takara, Dalian, China) for $30 \mathrm{~min}$ at $37^{\circ} \mathrm{C}$. Intracellular DNA was labeled with propidium iodide $\left(50 \mu \mathrm{g} / \mathrm{ml}\right.$; Sigma-Aldrich; Merck KGaA) at $4^{\circ} \mathrm{C}$ for $30 \mathrm{~min}$ and then analyzed using BD FACSCalibur flow cytometry (BD Bioscience, San Jose, CA, USA). The proportions of cells in the $\mathrm{G} 0 / \mathrm{G} 1, \mathrm{~S}$, and $\mathrm{G} 2 / \mathrm{M}$ phases were calculated using the ModFit software (Verity Software House Inc., Topsham, ME, USA).

Reverse transcription-quantitative polymerase chain reaction $(R T-q P C R)$. After transfection, total RNA were prepared by TRIzol extraction (Invitrogen; Thermo Fisher Scientific, Inc.) and reversed transcribed into cDNA using a PrimeScript RT reagent kit (Takara). RT-qPCR analyses were performed with SYBR-Green (Takara). Results were normalized to the expression of glyceraldehyde-3-phosphate dehydrogenase (GAPDH). Relative gene expression levels were calculated using the $2^{-\Delta \Delta C q}$ method (20).

Luciferase reporter assay. Cells were co-transfected with plasmid containing target genes [wild type (WT)- or mutant (Mut)-CASC15 and WT- or Mut-LGR5-3'UTR)] and $50 \mathrm{nM}$ miR-4310 mimic. Forty-eight hours after transfection, cells were collected and luciferase activity was detected by 

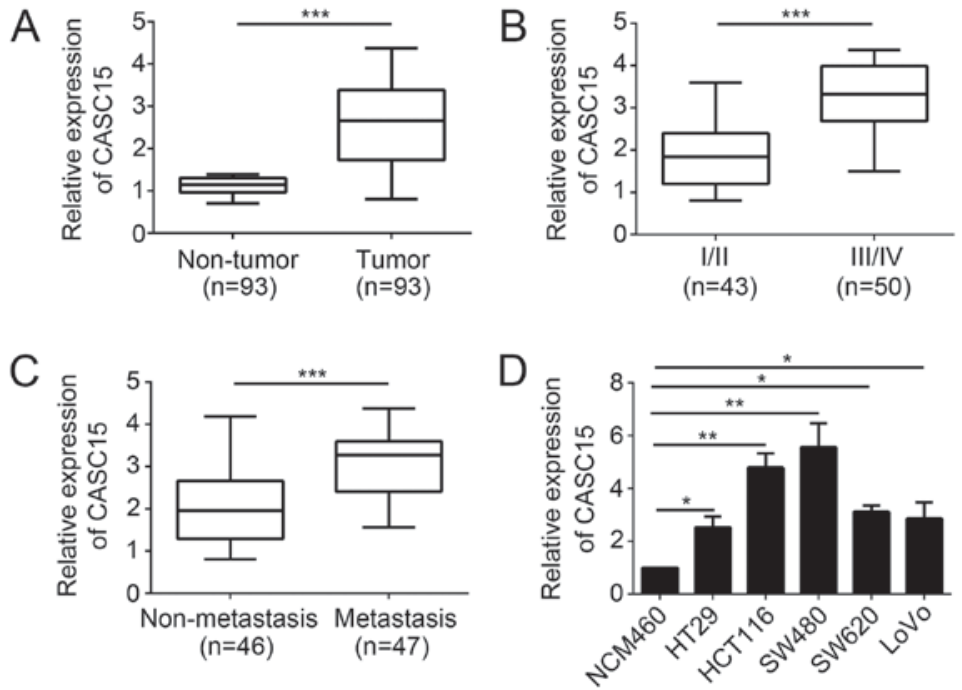

Figure 1. Expression of CASC15 is upregulated in CC tissues. (A) CASC15 expression was measured by RT-qPCR assay and normalized to GAPDH in 93 pairs of CC tissues and corresponding non-tumor tissues. (B and C) Comparisons of CASC15 levels in (B) CC patients at different stages (I/II stage, n=43; III/IV stage, $n=50)$ and $(C)$ with $(n=46)$ or without $(n=47)$ lymphatic metastasis. (D) The expression of CASC15 was measured in the CC cell lines and NCM460 cells by RT-qPCR. All data were obtained from three independent experiments and are presented as the mean \pm standard deviation. ${ }^{*} \mathrm{P}<0.05$, ${ }^{* *} \mathrm{P}<0.01$ and ${ }^{* * * *} \mathrm{P}<0.01$ vs. the control group (as indicated). CASC15, cancer susceptibility 15; CC, colon cancer; RT-qPCR, reverse transcription-quantitative polymerase chain reaction; GAPDH, glyceraldehyde-3-phosphate dehydrogenase.

Dual-Luciferase Reporter Assay kit (Promega Corp., Madison, WI, USA) in accordance with manufacturer's instructions.

Statistical analysis. All statistical analyses were performed using SPSS 20.0 (IBM Corp., Armonk, NY, USA) and GraphPad Prism version 5.0 software (GraphPad Software, Inc., La Jolla, CA, USA). Student's t-test and one-way analysis of variance followed by Tukey's post hoc test were used to analyze 2 or multiple groups, respectively, for statistical significance. Pearson correlation coefficient analysis was used to determine the correlations. $\mathrm{P}<0.05$ was considered to indicate a statistically significant difference.

\section{Results}

The expression of CASC15 was upregulated in CC tissues. We firstly analyzed the expression of CASC15 in CC tissues by RT-qPCR. The results showed that CASC15 was overexpressed in $\mathrm{CC}$ tissues compared to matched normal tissues (Fig. 1A; $\mathrm{P}<0.05$ ). Then we analyzed the correlation between CASC15 expression and clinicopathological features in CC patients. We divided these $\mathrm{CC}$ samples into two groups based on TNM stage. Through RT-qPCR, we found that the expression of CASC15 was higher in CC samples of stage III/IV than I/II (Fig. 1B; P<0.05). Moreover, the expression of CASC15 in metastatic $\mathrm{CC}$ patients was upregulated compared to those non-metastatic patients (Fig. $1 \mathrm{C} ; \mathrm{P}<0.05$ ). We then analyzed the expression of CASC15 in CC cell lines. RT-qPCR results indicated that CASC15 expression was higher in CC cell lines (HT29, HCT116, SW480, SW620, and LoVo) than in the human normal colonic epithelial cell line NCM460 (Fig. 1D; $\mathrm{P}<0.05)$. These data indicated that CASC15 was overexpressed in CC cells.

CASC15 knockdown inhibited cell proliferation in CC. Among all CC cell lines, the expression of CASC15 was highest in HCT116 and SW480 cells. Thus, we chose these two cell lines for experiments. To determine the function of CASC15, we knocked down CASC15 in HCT116 and SW480 cells. RT-qPCR results showed that the expression of CASC15 was significantly down-regulated in HCT116 and SW480 cells transfected shCASC15 (Fig. 2A; P<0.05). Then we performed CCK8 assay to evaluate cellular proliferation. As shown in Fig. 2B, knockdown of CASC15 significantly inhibited cellular proliferation. Moreover, FACS analysis indicated less HCT116 and SW480 cells entered into $S$ phase and G2/M phase after transfection with shCASC15, and more shCASC15 cells were arrested in $\mathrm{G} 0 / \mathrm{G} 1$ phase (Fig. $2 \mathrm{C}$; $\mathrm{P}<0.05$ ). In consistence, knockdown of CASC15 decreased the protein levels of Cyclin D1 and Ki67 in HCT116 and SW480 cells (Fig. 2D; $\mathrm{P}<0.05)$, which suggested that CASC15 knockdown inhibited $\mathrm{CC}$ cell proliferation.

CASC15 knockdown repressed cell migration and invasion. We above demonstrated a positive correlation between CASC15 expression and tumor metastasis. We then checked the effect of CASC15 on cell migration and invasion. Through Transwell assay, we found that knockdown of CASC15 significantly suppressed the migration and invasion of HCT116 and SW480 cells (Fig. 3A and B). Moreover, the expression of metastasis-related proteins, Twist and Snail, was significantly downregulated in HCT116 and SW480 cells transfected with shCASC15 (Fig. 3C).

CASC15 promoted CC cell proliferation, migration and invasion throughregulation ofmiR-4310/LGR5/Wnt/ $\beta$-catenin pathway. LncRNAs have been demonstrated to serve as competing endogenous RNAs for miRNAs. Thus, we analyzed the predicted target miRNAs of CASC 15 by informatics analysis. Among all potential targets, miR-4310 ranked first. We found that there were two potential binding sites of miR-4310 in CASC15 (Fig. 4A). Luciferase reporter 

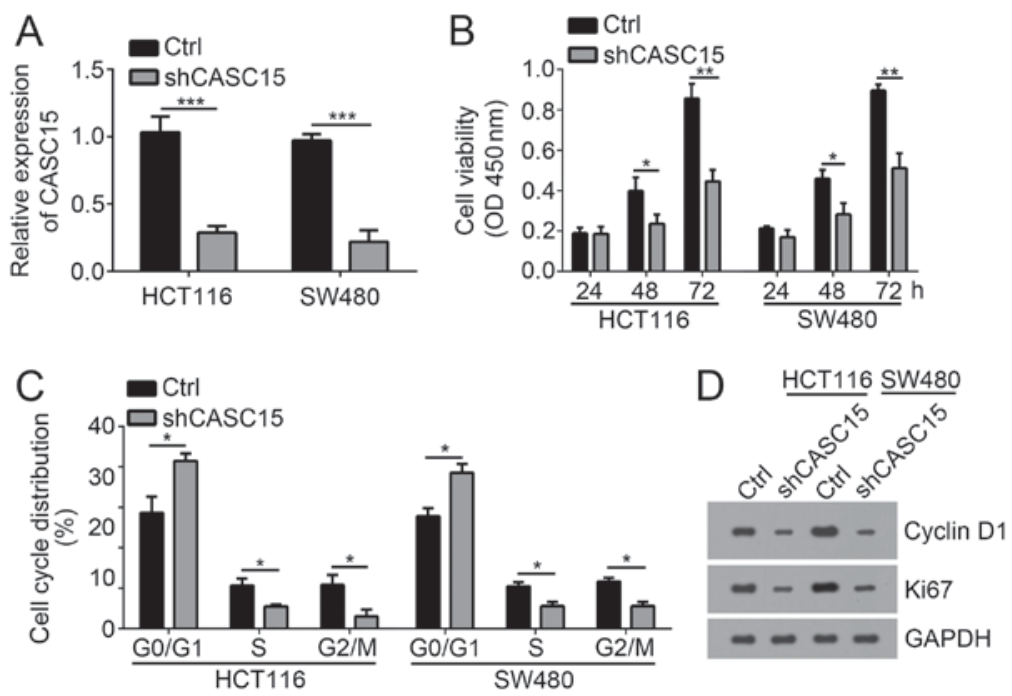

Figure 2. CASC15 knockdown inhibits cell proliferation in CC. (A) Inhibitory efficiency of shCASC15 on CASC15 expression in HCT116 and SW480 cells was evaluated by reverse transcription-quantitative polymerase chain reaction assay. (B) HCT116 and SW480 cells were transfected with shCASC15 or Ctrl plasmids. Then Cell Counting kit- 8 assay was performed to determine cell viability at 24,48 and $72 \mathrm{~h}$ following transfection. (C) Flow cytometry analysis was used for cell cycle evaluation. (D) The protein levels of Ki67 and Cyclin D1 were measured by western blotting in HCT116 and SW480 cells transfected with shCASC15 or Control. All data were obtained from three independent experiments and are presented as the mean \pm standard deviation. "P<0.05, ${ }^{* *} \mathrm{P}<0.01$ and ${ }^{* * *} \mathrm{P}<0.01$ vs. the Ctrl group (as indicated). Ctrl, control; CASC15, cancer susceptibility $15 ; \mathrm{CC}$, colon cancer; sh-, short hairpin RNA; GAPDH, glyceraldehyde-3-phosphate dehydrogenase.
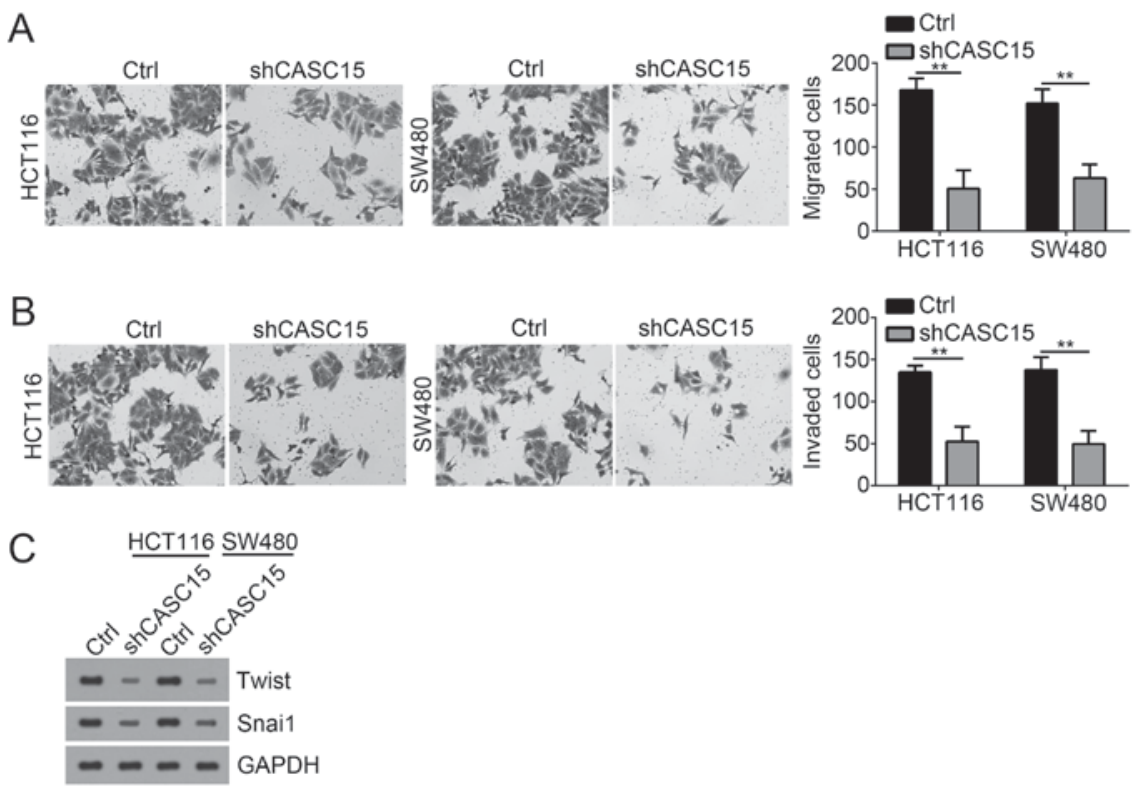

Figure 3. CASC15 knockdown represses cell migration and invasion. (A and B) Transwell assay was used to analyze the (A) migration and (B) invasion of HCT116 and SW480 cells transfected with shCASC15 or Control (magnification, x100). (C) The protein levels of Twist and Snail were measured by western blotting in HCT116 and SW480 cells transfected with shCASC15 or Control. All data were obtained from three independent experiments and are presented as the mean \pm standard deviation. ${ }^{* *} \mathrm{P}<0.01$ vs. the Ctrl group (as indicated). Snail, Snail family transcriptional repressor 1; Ctrl, control; CASC15, cancer susceptibility 15; sh-, short hairpin RNA; GAPDH, glyceraldehyde-3-phosphate dehydrogenase.

assay indicated that overexpression of miR-4310 repressed the luciferase intensity in HCT116 and SW480 cells transfected with WT-CASC15 reporter vector (Fig. 4B). Moreover, overexpression of miR-4310 significantly suppressed the expression of CASC15 (Fig. 4C). MiRNAs could target the complementary sequence of target mRNAs to regulate gene expression. To further explore the downstream signal, we conducted another prediction and found that LGR5 was a potential target of mIR-4310. There was a complementary sequence of miR-4310 in the 3'-UTR region of LGR5 mRNA (Fig. 4D). Similarly, luciferase reporter assay indicated that overexpression of miR-4310 significantly inhibited the luciferase activity in HCT116 and SW480 cells transfected with WT-LGR5 reporter vector (Fig. 4E). And overexpression of miR-4310 suppressed the mRNA and protein level of LGR5 (Fig. 4F and G). LGR5 is acknowledged as an essential regulator for activation of $\mathrm{Wnt} / \beta$-catenin pathway $(21,22)$. Then we determined whether CASC15 
A

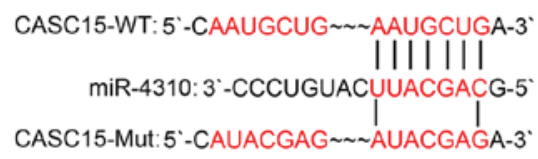

D

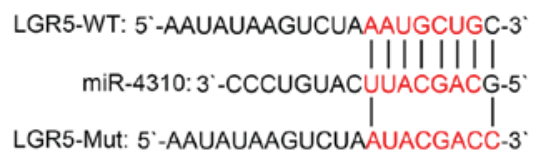

B

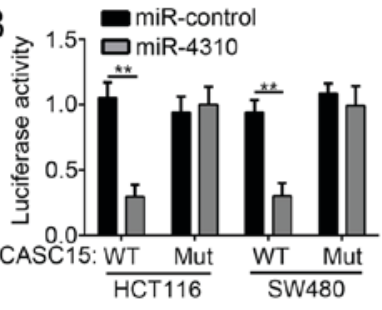

E

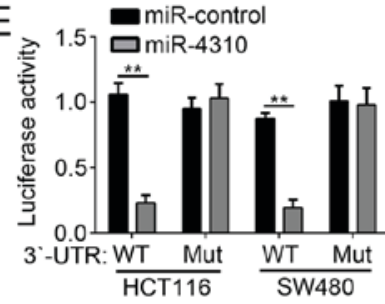

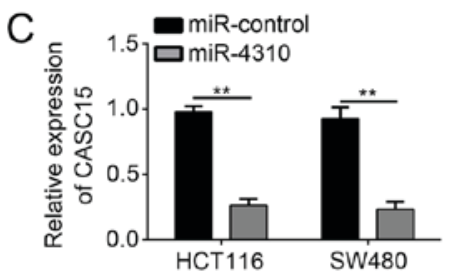

F

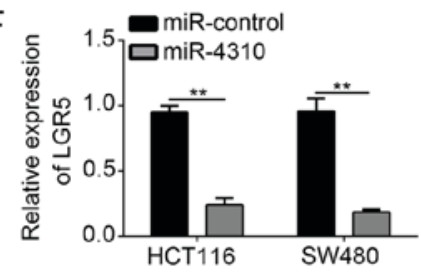

G
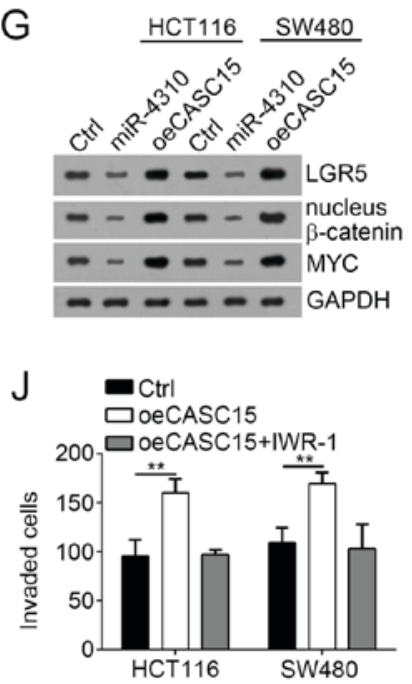

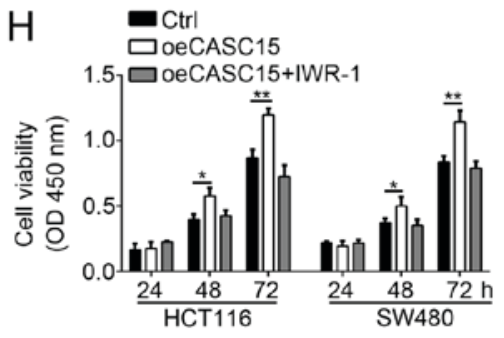

I

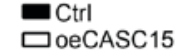

250 口0eCASC15+IWR-1

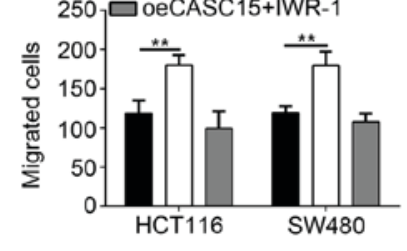

Figure 4. CASC15 promotes CC cell proliferation, migration and invasion through the regulation of the miR-4310/LGR5/Wnt/ $\beta$-catenin signaling pathway. (A) Predicted binding sites in CASC15 with miR-4310. (B) Luciferase reporter assay indicated that overexpression of miR-4310 suppressed the luciferase intensity in HCT116 and SW480 cells transfected with WT CASC15 reporter. (C) Overexpression of miR-4310 reduced the expression of CASC15 in HCT116 and SW480 cells. (D) Predicted complementary site of miR-4310 in 3'-UTR region of LGR5 mRNA. (E) Luciferase activity assay revealed that overexpression of miR-4310 reduced the luciferase activity in HCT116 and SW480 cells transfected with LGR5-WT-3'-UTR reporter. (F) Overexpression of mIR-4310 inhibited the mRNA level of LGR5 in HCT116 and SW480 cells. (G) Western blotting demonstrated that the LGR5 protein level and activation of Wnt/ $\beta$-catenin were enhanced by CASC15 overexpression, but was impaired by miR-4310 overexpression in HCT116 and SW480 cells. (H) Cell Counting kit- 8 assay was used to assess the proliferation of the indicated cell lines. (I and J) Inhibition of the Wnt/ $\beta$-catenin signaling pathway reversed the effects of CASC15 overexpression on CC cell (I) migration and ( $\mathrm{J}$ ) invasion. All data were obtained from three independent experiments and are presented as the mean \pm standard deviation. ${ }^{*} \mathrm{P}<0.05$ and ${ }^{* *} \mathrm{P}<0.01$ vs. the control group (as indicated). UTR, untranslated region; Ctrl, control; CASC15, cancer susceptibility 15; CC, colon cancer; sh-, short hairpin RNA; miR, microRNA; WT, wild type; Mut, mutant; LGR5, leucine-rich repeat-containing G-protein coupled receptor 5; oe-, overexpression; GAPDH, glyceraldehyde-3-phosphate dehydrogenase.

regulated Wnt/ $\beta$-catenin pathway by LGR5. Western blot results indicated that overexpression of CASC15 significantly upregulated the expression of LGR5 and increased the protein level of nucleus $\beta$-catenin and MYC (Fig. 4G), which suggested that CASC15 promoted the activation of Wnt/ $\beta$-catenin. To further determine whether CASC15 promotes $\mathrm{CC}$ cell proliferation, migration and invasion in a Wnt $/ \beta$-catenin pathway dependent manner, we inhibited the Wnt/ $\beta$-catenin pathway with a specific inhibitor (IWR-1) in CASC15-overexpressed HCT116 and SW480 cells. Then we performed functional experiments. Through CCK8 and Transwell assay, we found that overexpression of CASC15 promoted the proliferation, migration and invasion of $\mathrm{CC}$ cells while inhibition of $\mathrm{Wnt} / \beta$-catenin pathway reversed CASC15-mediated effects (Fig. 4H-J). Taken together, our results indicated that $\mathrm{Wnt} / \beta$-catenin pathway is important for the function of CASC15 in CC.

CASC15 knockdown reduced tumor growth in vivo. We further investigated the function of CASC15 in CC cells in vivo. We subcutaneously injected shCASC15 or control SW480 cells into the flank of nude mice. We measured tumor volumes at indicative time points. As shown in Fig. 5A, knockdown of CASC15 significantly inhibited tumor growth in vivo. Moreover, the tumor tissues in shCASC15 group were smaller than control group (Fig. 5B). Then we measured the protein levels of proliferation- or metastasis-related genes in form tumor tissues. We found that knockdown of CASC15 significantly inhibited the levels of Cyclin D1, Ki67, Twist, Snail and LGR5 (Fig. 5C). These data indicated that CASC15 promoted CC progression in vivo. 

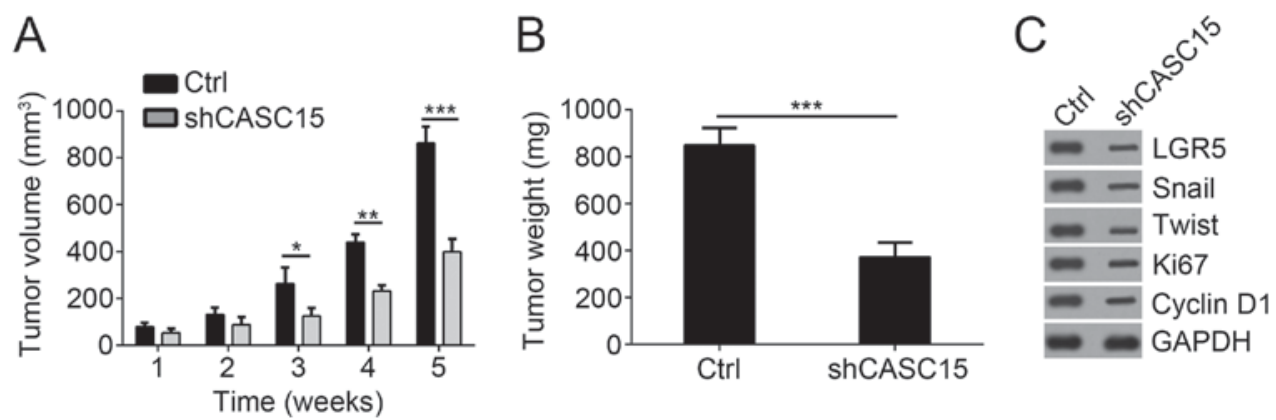

Figure 5. CASC15 knockdown reduces tumor growth in vivo. (A) The volumes in subcutaneous xenografts were measured and calculated once a week for 5 weeks. (B) Tumor weight was measured at the end of the experiments. (C) Protein levels of Cyclin D1, Ki67, Twist, Snail and LGR5 were measured by western blotting in formed tumor tissues. All data were obtained from three independent experiments and are presented as the mean \pm standard deviation. $\mathrm{P}<0.05$, ${ }^{* *} \mathrm{P}<0.01$ and ${ }^{* * * *} \mathrm{P}<0.001$ vs. the control group (as indicated). Ctrl, control; CASC15, cancer susceptibility 15; sh-, short hairpin RNA; LGR5, leucine-rich repeat-containing G-protein coupled receptor 5; GAPDH, glyceraldehyde-3-phosphate dehydrogenase.

\section{Discussion}

$\mathrm{CC}$ is one of the highly lethal cancers worldwide and the incidence is gradually increasing year by year (23). Dysregulation of lncRNA is closely related with tumor growth and metastasis in various cancers (24). Therefore, identifying important lncRNAs involved in CC will benefit for CC intervention. In this study, we identified that CASC15 was highly expressed in $\mathrm{CC}$ tissues and cell lines compared to normal control. Moreover, we found that the expression of CASC15 was positively correlated with CC TNM stage and metastasis, which suggested that CASC15 may serve as a promising prognostic biomarker and therapeutic target for $\mathrm{CC}$ treatment.

LncRNAs belong to one of non-coding RNAs, including microRNA, housekeeping ncRNA, and regulatory ncRNA (25). Notably, lncRNAs could act as invasive and sensitive molecular markers for the screening and early diagnosis in various cancers $(25,26)$. In the past decade, IncRNAs have been a very hot topic in the field of tumor biology. Increasing evidence indicates that IncRNAs play essential roles in the development and progression of various cancers through regulating tumor cell survival, growth and metastasis $(27,28)$. For example, the lncRNA SNHG3 functions as a competing endogenous RNA to promote malignant development of colorectal cancer (29). The noncoding RNA HOXD-AS1 is a critical regulator of the metastasis and apoptosis phenotype in human hepatocellular carcinoma (30). LncRNA linc00673 regulated non-small cell lung cancer proliferation, migration, invasion and epithelial mesenchymal transition by sponging miR-150-5p (31). Previous study indicated that high expression of IncRNA CASC15 is a risk factor for gastric cancer prognosis and promote the proliferation of gastric cancer (32). In addition, a study showed that CASC15 is involved in melanoma progression and phenotype-switching (33). And another report indicated that CASC15 promotes RUNX1-rearranged acute leukemia by regulating SOX4 expression (34). However, the function of CASC15 in CC remains largely unknown. In our study, we found that knockdown of CASC15 significantly inhibited proliferation, migration and invasion on CC cells in vitro and in vivo. And CASC15 knockdown inhibited the expression of Twist and Snail. Our findings demonstrated that CASC15 also serves as an oncogene in CC and suggested that CASC15 might be a potential therapeutic target for $\mathrm{CC}$ treatment.
Wnt/ $\beta$-catenin signaling pathway has been acknowledged as an essential regulator in cellular proliferation (35). Abnormal activation of $\mathrm{Wnt} / \beta$-catenin signaling pathway is linked to occurrence of various cancers (36). Accumulating evidence indicates that $\mathrm{Wnt} / \beta$-catenin signaling pathway plays a vital role on the regulation of tumor growth and metastasis in cancers, including CC. A study showed that the WNT signaling pathway was activated in $93 \%$ of non-hypermutated CRCs and $97 \%$ of hypermutated colorectal cancer (37). For instance, Yang et al (38) reported that microRNA-320 regulates the radiosensitivity of cervical cancer cells C33AR by targeting $\beta$-catenin. Qi et al (39) showed that Wnt3a promotes the vasculogenic mimicry formation of $\mathrm{CC}$ via Wnt/ $\beta$-catenin signaling. Besides, another report indicated that new Wnt/ $\beta$-catenin target genes promote experimental metastasis and migration of colorectal cancer cells through different signals (40). The significance of $\mathrm{Wnt} / \beta$-catenin pathway in $\mathrm{CC}$ has been emphasized in the past decades. Therefore, uncovering the regulatory mechanism of Wnt/ß-catenin pathway is especially important. In our study, we found that CASC15 served as a sponge of miR-4310 and consequently promoted the expression of LGR5. Upregulated LGR5 expression eventually activated Wnt/ $\beta$-catenin pathway in CC cells. Through luciferase reporter assay, we validated the direct interaction between miR-4310 and CASC15 or LGR5 3'UTR. We also demonstrated that CASC15 overexpression promoted LGR5 expression by inhibiting miR-4310 availability. By western blot, we found that increased level of LGR5 led to more nucleus $\beta$-catenin and MYC expression in $\mathrm{CC}$ cells, indicating activation of $\beta$-catenin signaling pathway. Moreover, we found that inhibition of $\beta$-catenin pathway abolished the elevating effects on CC cell proliferation, migration and invasion by CASC15.

In conclusion, our finding demonstrated that CASC15 promoted the proliferation, migration and invasion of $\mathrm{CC}$ cells through regulation of miR-4310/LGR5/Wnt/ $\beta$-catenin axis. Our results suggested that CASC15 might serve as a biomarker for diagnosis and prognosis, and a therapeutic target for the treatment of CC patients.

\section{Acknowledgements}

Not applicable. 


\section{Funding}

No funding was received.

\section{Availability of data and materials}

All data generated or analyzed during this study are included in this published article.

\section{Authors' contributions}

NJ, TH and YZ conceived and designed the present study, analyzed and interpreted the results, and wrote the manuscript. HG, JY, ML and ZC performed the experiments. All authors read and approved the final manuscript.

\section{Ethics approval and consent to participate}

The present study was approved by the Institutional Ethics Committee of Tumor Hospital of Jilin Province; all enrolled patients provided written informed consent. Animal studies were approved by the Medical Experimental Animal Care Commission at Tumor Hospital of Jilin Province.

\section{Patient consent for publication}

All patients provided written informed consent for the publication of their data.

\section{Competing interests}

The authors declare that they have no competing interests.

\section{References}

1. Siegel RL, Miller KD and Jemal A: Cancer statistics, 2017. CA Cancer J Clin 67: 7-30, 2017.

2. Chen W, Zheng R, Baade PD, Zhang S, Zeng H, Bray F, Jemal A Yu XQ and He J: Cancer statistics in China, 2015. CA Cancer J Clin 66: 115-132, 2016

3. Chen W, Zheng R, Zhang S, Zhao P, Zeng H and Zou X: Report of cancer incidence and mortality in China, 2010. Ann Trans Med 2: 61, 2014

4. Lee YC, Lee YL, Chuang JP and Lee JC: Differences in survival between colon and rectal cancer from SEER data. PLoS One 8 : e78709, 2013

5. Kapranov P,Cheng J,Dike S, Nix DA, DuttaguptaR, Willingham AT, Stadler PF, Hertel J, Hackermüller J, Hofacker IL, et al: RNA maps reveal new RNA classes and a possible function for pervasive transcription. Science 316: 1484-1488, 2007.

6. Ponting CP, Oliver PL and Reik W: Evolution and functions of long noncoding RNAs. Cell 136: 629-641, 2009.

7. Guttman M, Garber M, Levin JZ, Donaghey J, Robinson J, Adiconis X, Fan L, Koziol MJ, Gnirke A, Nusbaum C, et al: $\mathrm{Ab}$ initio reconstruction of cell type-specific transcriptomes in mouse reveals the conserved multi-exonic structure of lincRNAs Nat Biotechnol 28: 503-510, 2010.

8. Ye B, Liu B, Yang L, Zhu X, Zhang D, Wu W, Zhu P, Wang Y, Wang S, Xia P, et al: LncKdm2b controls self-renewal of embryonic stem cells via activating expression of transcription factor Zbtb3. EMBO J 37: pii: e97174, 2018.

9. Fu Q, Qin Z, Zhang L, Lyu D, Tang Q, Yin H, Chen Z and Yao K: A new long noncoding RNA ALB regulates autophagy by enhancing the transformation of LC3BI to LC3BII during human lens development. Mol Ther Nucleic Acids 9: 207-217, 2017.

10. Liu B, Ye B, Yang L, Zhu X, Huang G, Zhu P, Du Y, Wu J, Qin X, Chen $\mathrm{R}$, et al: Long noncoding RNA $\operatorname{lncKdm} 2 \mathrm{~b}$ is required for ILC3 maintenance by initiation of Zfp292 expression. Nat Immunol 18: 499-508, 2017.
11. Li J, Wu QM, Wang XQ and Zhang CQ: Long noncoding RNA miR210HG sponges miR-503 to facilitate osteosarcoma cell invasion and metastasis. DNA Cell Biol 36: 1117-1125, 2017.

12. Alipour A, Mojdehfarahbakhsh A, Tavakolian A, Morshedzadeh T, Asadi M, Mehdizadeh A and Nami M: Neural communication through theta-gamma cross-frequency coupling in a bistable motion perception task. J Integr Neurosci 15: 539-551, 2016.

13. Wang J, Ma W and Liu Y: Long non-coding RNA HULC promotes bladder cancer cells proliferation but inhibits apoptosis via regulation of ZIC2 and PI3K/AKT signaling pathway. Cancer Biomark 20: 425-434, 2017.

14. Gong W, Tian M, Qiu H and Yang Z: Elevated serum level of lncRNA-HIF1A-AS1 as a novel diagnostic predictor for worse prognosis in colorectal carcinoma. Cancer Biomark 20: 417-424, 2017.

15. Ma Z, Gu S, Song M, Yan C, Hui B, Ji H, Wang J, Zhang J, Wang K and Zhao Q: Long non-coding RNA SNHG17 is an unfavourable prognostic factor and promotes cell proliferation by epigenetically silencing P57 in colorectal cancer. Mol Biosyst 13: 2350-2361, 2017.

16. Bao H, Guo CG, Qiu PC, Zhang XL, Dong Q and Wang YK: Long non-coding RNA Igf2as controls hepatocellular carcinoma progression through the ERK/MAPK signaling pathway. Oncol Lett 14: 2831-2837, 2017.

17. Su J, Zhang E, Han L, Yin D, Liu Z, He X, Zhang Y, Lin F, Lin Q, Mao P, et al: Long noncoding RNA BLACAT1 indicates a poor prognosis of colorectal cancer and affects cell proliferation by epigenetically silencing of p15. Cell Death Dis 8: e2665, 2017.

18. Zhao Z, Wang J, Wang S, Chang H, Zhang T and Qu J: LncRNA CCAT2 promotes tumorigenesis by over-expressed Pokemon in non-small cell lung cancer. Biomed Pharmacother 87: 692-697, 2017.

19. He T, Zhang L, Kong Y, Huang Y, Zhang Y, Zhou D, Zhou X, Yan Y, Zhang L, Lu S, et al: Long non-coding RNA CASC15 is upregulated in hepatocellular carcinoma and facilitates hepatocarcinogenesis. Int J Oncol 51: 1722-1730, 2017.

20. Livak KJ and Schmittgen TD: Analysis of relative gene expression data using real-time quantitative PCR and the 2(-Delta Delta C(T)) method. Methods 25: 402-408, 2001.

21. Lin YU, Wu T, Yao Q, Zi S, Cui L, Yang M and Li J: LGR5 promotes the proliferation of colorectal cancer cells via the Wnt $/ \beta$-catenin signaling pathway. Oncol Lett 9: 2859-2863, 2015 .

22. Carmon KS, Lin Q, Gong X, Thomas A and Liu Q: LGR5 interacts and cointernalizes with Wnt receptors to modulate Wnt/ß-catenin signaling. Mol Cell Biol 32: 2054-2064, 2012.

23. Torre LA, Bray F, Siegel RL, Ferlay J, Lortet-Tieulent J and Jemal A: Global cancer statistics, 2012. CA Cancer J Clin 65 87-108, 2015.

24. Shang W, Tang Z, Gao Y, Qi H, Su X, Zhang Y and Yang R: LncRNA RNCR3 promotes Chop expression by sponging miR-185-5p during MDSC differentiation. Oncotarget 8: $111754-111769,2017$

25. Inamura K: Major tumor suppressor and oncogenic non-coding RNAs: Clinical relevance in lung cancer. Cells 6: pii: E12, 2017.

26. Inamura $\mathrm{K}$ and Ishikawa Y: MicroRNA in lung cancer: Novel biomarkers and potential tools for treatment. J Clin Med 5: pii: E36, 2016.

27. Cao Y, Shi H, Ren F, Jia Y and Zhang R: Long non-coding RNA CCAT1 promotes metastasis and poor prognosis in epithelial ovarian cancer. Exp Cell Res 359: 185-194, 2017.

28. Wang Y, Kuang H, Xue J, Liao L, Yin F and Zhou X: LncRNA AB073614 regulates proliferation and metastasis of colorectal cancer cells via the PI3K/AKT signaling pathway. Biomed Pharmacother 93: 1230-1237, 2017.

29. Huang W, Tian Y, Dong S, Cha Y, Li J, Guo X and Yuan X: The long non-coding RNA SNHG3 functions as a competing endogenous RNA to promote malignant development of colorectal cancer. Oncol Rep 38: 1402-1410, 2017.

30. Lu S, Zhou J, Sun Y, Li N, Miao M, Jiao B and Chen H: The noncoding RNA HOXD-AS1 is a critical regulator of the metastasis and apoptosis phenotype in human hepatocellular carcinoma. Mol Cancer 16: 125, 2017.

31. Lu W, Zhang H, Niu Y, Wu Y, Sun W, Li H, Kong J, Ding K, Shen HM, Wu H, et al: Long non-coding RNA linc00673 regulated non-small cell lung cancer proliferation, migration, invasion and epithelial mesenchymal transition by sponging miR-150-5p. Mol Cancer 16: 118, 2017. 
32. Yao XM, Tang JH, Zhu $\mathrm{H}$ and Jing Y: High expression of LncRNA CASC15 is a risk factor for gastric cancer prognosis and promote the proliferation of gastric cancer. Eur Rev Med Pharmacol Sci 21: 5661-5667, 2017.

33. Lessard L, Liu M, Marzese DM, Wang H, Chong K, Kawas N, Donovan NC, Kiyohara E, Hsu S, Nelson N, et al: The CASC15 long intergenic noncoding RNA locus is involved in melanoma progression and phenotype switching. J Invest Dermatol 135: 2464-2474, 2015.

34. Fernando TR, Contreras JR, Zampini M, Rodriguez-Malave NI, Alberti MO, Anguiano J, Tran TM, Palanichamy JK, Gajeton J, Ung NM, et al: The lncRNA CASC15 regulates SOX4 expression in RUNX1-rearranged acute leukemia. Mol Cancer 16: 126, 2017.

35. Liang S, Zhang S, Wang P, Yang C, Shang C, Yang J and Wang J: LncRNA, TUG1 regulates the oral squamous cell carcinoma progression possibly via interacting with $\mathrm{Wnt} / \beta$-catenin signaling. Gene 608: 49-57, 2017.

36. Guan H, Liu C, Fang F, Huang Y, Tao T, Ling Z, You Z, Han X, Chen S, Xu B and Chen M: MicroRNA-744 promotes prostate cancer progression through aberrantly activating Wnt/ $\beta$-catenin signaling. Oncotarget 8: 14693-14707, 2017.
37. Inamura K: Colorectal cancers: An update on their molecular pathology. Cancers (Basel) 10: pii: E26, 2018.

38. Yang CX, Zhang SM, Li J, Yang B, Ouyang W, Mei ZJ, Chen J, Dai J, Ke S, Zhou FX, et al: MicroRNA-320 regulates the radiosensitivity of cervical cancer cells C33AR by targeting $\beta$-catenin. Oncol Lett 12: 4983-4990, 2016.

39. Qi L, Song W, Liu Z, Zhao X, Cao W and Sun B: Wnt3a promotes the vasculogenic mimicry formation of colon cancer via Wnt/ $\beta$-catenin signaling. Int J Mol Sci 16: 18564-18579, 2015.

40. Qi J, Yu Y, Akilli Öztürk Ö, Holland JD, Besser D, Fritzmann J, Wulf-Goldenberg A, Eckert K, Fichtner I and Birchmeier W: New Wnt/ $\beta$-catenin target genes promote experimental metastasis and migration of colorectal cancer cells through different signals. Gut 65: 1690-1701, 2016. 